\title{
MANEJO RESPONSABLE DEL MERCURIO DE LA AMALGAMA DENTAL: UNA REVISIÓN SOBRE SUS REPERCUSIONES EN LA SALUD
}

\author{
Christhian Méndez-Visag ${ }^{1, a}$
}

\begin{abstract}
RESUMEN
La presente revisión trata sobre las repercusiones del empleo de la amalgama dental en el trabajo odontológico. El documento se enfoca por un lado en la situación del feto quien es el más vulnerable a la exposición del mercurio de la amalgama dental materna, por la forma crónica y la magnitud a la que puede ser expuesto; por otro lado, se aborda la toma de conciencia de las repercusiones medioambientales que conlleva la continuidad en el empleo de este material restaurador cuando no se cumplen con las mejores prácticas de manejo (MPM) impulsadas por la OMS y la Asociación Dental Americana sobre el manejo del mercurio, que es un químico persistente, bioacumulable y toxico. Pese a que la amalgama dental, se encuentra en pleno declive en cuanto a su empleo en el sector público y privado, es necesario que se pongan en práctica las medidas difundidas por la OMS respecto a las MPM por parte del profesional odontólogo y por las instituciones académicas que podrían seguir enseñando su empleo, es necesario fomentar desde el pregrado los valores éticos de responsabilidad con la salud y el medioambiente considerando que el pobre manejo del mercurio contribuye a la carga global de mercurio ambiental. Finalmente, las conclusiones aportan modificaciones importantes tanto en el campo clínico, el principio de precaución, y el aspecto logístico de la profesión en el proceso de reducir, hasta llegar a la eliminación completa, el uso del mercurio.
\end{abstract}

Palabras clave: Mercurio; Toxicidad; Contaminación; Costo efectividad (fuente: DeCS BIREME).

\section{RESPONSIBLE MANAGEMENT OF DENTAL AMALGAM MERCURY: A REVIEW OF ITS IMPACT ON HEALTH}

\begin{abstract}
This review discusses the implications of dental amalgam used in dentistry. We first focus on the status of the fetus, which is the most vulnerable to mercury exposure from maternal dental amalgams because of the chronic form and potential magnitude of exposure. And second, our work covers the awareness of environmental repercussions involved with continued use of this restorative material, a persistent, bioaccumulative and toxic chemical, when best management practices (BMPs) of mercury from the WHO and the American Dental Association are not followed. Although the use of dental amalgam is in decline in the public and private sectors, it is necessary that the measures disseminated by the WHO on BMPs are implemented by professional dentists and taught by academic institutions that may continue to teach its use. It is also essential to promote from the undergraduate level the ethical values and responsibility to health and the environment, considering that the poor handling of mercury contributes to the global burden of environmental mercury. Finally, the findings support important modifications in the clinical field, the principle of precaution, and logistical aspects of the profession in the process of reducing and eventually eliminating the use of mercury.
\end{abstract}

Key words: Mercury; Toxicity; Contamination; Cost effectiveness (source: MeSH, NLM).

\section{INTRODUCCIÓN}

La amalgama dental es la principal fuente de mercurio en la atención de salud, y es el mayor contribuyente en la carga corporal de dicho metal en personas portadoras de estas restauraciones (1), mientras que en los no portadores es el pescado la fuente común de mercurio en la población (2). La amalgama dental se ha usado desde el siglo XIX como uno de los mejores materiales de restauración, no solo por su durabilidad y adaptabilidad en cavidades dentarias posteriores, sino por su "costoefectividad" (3). Se enfatiza este último aspecto derivado de evaluaciones económicas, debido a que se demostró que la amalgama dental es el material más costoso entre los materiales de restauración dental, si los costos ambientales se incluyen en el cálculo económico ${ }^{(4,5)}$.

\footnotetext{
Universidad Nacional Mayor de San Marcos. Lima, Perú.

a Cirujano dentista

Recibido: 07-05-14 Aprobado: 20-08-14
}

Citar como: Méndez-Visag C. Manejo responsable del mercurio de la amalgama dental: una revisión sobre sus repercusiones en la salud. Rev Peru Med Exp Salud Publica. 2014;31(4):725-32. 
La metodología empleada en la presente revisión tuvo como criterios de selección de artículos los términos de búsqueda: amalgama dental, toxicidad, mercurio y salud pública, en las bases de datos Scielo y Redalyc; y los términos mencionados en inglés en las bases de datos PubMed, HINARI, EBSCO, ScienceDirect y SCOPUS, disponibles de diciembre de 2013 a febrero de 2014. El objetivo fue presentar la información científica más relevante sobre las repercusiones de la toxicidad del mercurio en la salud maternoinfantil y en el medioambiente; así como las medidas de salud pública adoptadas por diferentes países.

\section{EVIDENCIA CIENTIIFICA DE LA TRANSFERENCIA DEL MERCURIO DE LA AMALGAMA DENTAL MATERNAAL FETO}

La importancia global de las amalgamas dentales, especialmente de la madre que contribuyen significativamente a la carga corporal de mercurio $(\mathrm{Hg})$ en el feto y en la infancia temprana, se deriva del hecho de que algunos estudios han postulado que la exposición al mercurio puede causar discapacidades del desarrollo neurológico en los bebés, disfunciones inmunológicas, sensorio neurológicas, motoras y de comportamiento, similares a los rasgos definidos 0 asociados con trastornos del espectro autista (TEA), y que estas similitudes se extienden a la neuroanatomía, neurotransmisores, y la bioquímica ${ }^{(6,7)}$.

La susceptibilidad de los niños a los efectos adversos por la exposición al $\mathrm{Hg}$ puede cambiar con el tiempo, dependiendo de la etapa de desarrollo. La primera exposición de los niños a los contaminantes ambientales se lleva a cabo durante el desarrollo prenatal a través de transporte transplacentario, ya que la placenta humana no representa un obstáculo real para el transporte de mercurio elemental $\left(\mathrm{Hg}^{0}\right)$ y metilmercurio $(\mathrm{MeHg}){ }^{(8)}$. Un incremento en la concentración de $\mathrm{Hg}$ en la sangre del cordón, en la placenta o la leche materna ${ }^{(9)}$ en relación con el número de restauraciones de amalgama materna se muestra en varios estudios. Por el contrario, otros autores han enfatizado en que la exposición al $\mathrm{Hg}$ de las restauraciones dentales de amalgama se encuentra en niveles bajos, y han argumentado que, a pesar de la exposición a largo plazo, no es suficiente para afectar negativamente la salud humana ${ }^{(10-12)}$. La mayoría de estos estudios se centró en la exposición de amalgama en las poblaciones de niños y adultos, pero no en fetos ni en recién nacidos ${ }^{(6)}$. Y, es justo antes del parto cuando los niños están más expuestos al metilmercurio y al mercurio inorgánico con respecto a los niños lactantes ${ }^{(13)}$.

Los niveles de $\mathrm{Hg}^{0}$ del aire en la boca se correlacionan significativamente con el número de superficies de amalgama debido a que pequeñas cantidades de $\mathrm{Hg}^{0}$ y mercurio inorgánico divalente $\left(\mathrm{Hg}^{2+}\right)$ que se liberan de forma continua desde la superficie de la restauración en la boca, sea por corrosión de la amalgama o movimientos de masticación. Estimaciones promedio de la cantidad de mercurio absorbido de las amalgamas dentales varían de 3 a $17 \mu \mathrm{g} / \mathrm{día}$, dependiendo del número total de las restauraciones dentales con ese material $(1,6,14,15)$. Los niveles de mercurio oscilan entre el límite de detección (LDD; 0,1 $\mu \mathrm{g} / \mathrm{L}$ ) a $780 \mu \mathrm{g} / \mathrm{L}$, en flujo salival basal en estado no estimulado y en una prueba posterior a la goma de mascar ${ }^{(14)}$. Más aun, se reportaron casos de bruxomanos y personas con hábito a la goma de mascar, cuya excreción de mercurio en orina estuvo en el rango de 25 a $54 \mu \mathrm{g} / \mathrm{g}$ creatinina, indicando una absorción diaria de hasta $100 \mu \mathrm{g}$ de mercurio. Estos valores equivalen a cinco o hasta diez veces el promedio en la población general ${ }^{(16,17)}$.

Pruebas sobre la difusión del mercurio desde la madre al feto se han determinado en estudios en humanos y apuntan a que el feto es expuesto a una dosis más alta de mercurio cuando la madre es sometida a restauraciones de amalgama, y mucho más expuesto cuando es sometida a la remoción de amalgamas antiguas ${ }^{(18,19)}$, ya que las concentraciones de vapor de mercurio en el aire de la zona del procedimiento puede alcanzar valores de $388 \mu \mathrm{g} / \mathrm{m}^{3}$ y de $1500 \mu \mathrm{g} /$ $\mathrm{m}^{3}$ respectivamente ${ }^{(9,15)}$. Además, se ha determinado que trazas de mercurio elemental e inorgánico de la saliva son tomadas por bacterias orales que, a su vez, liberan metilmercurio como producto (14). Estas bacterias son bacterias reductoras de sulfato (BRS) que se encuentra en la boca de aproximadamente el 10\% de los sujetos periodontalmente sanos; mientras que entre los pacientes con periodontitis, la frecuencia de presencia de BRS aumentó signicativamente (58-72\% de los pacientes) ${ }^{(20)}$.

Una vez ingerido en el tracto gastrointestinal, el metilmercurio en la saliva es, por tanto, casi totalmente absorbido (> 95\%). En un estudio el $15-18 \%$ de mercurio total en saliva (5-12,5 $\mathrm{nmol} / L$ ) fue orgánico en un grupo de sujetos con restauraciones de amalgama. Estos sujetos tenían, en promedio, 22 superficies de amalgama (rango, 2-51). En el grupo sin amalgama, el mercurio orgánico fue 2-5 nmol/L. La exposición al metilmercurio a través de la ingestión derivada de la biometilación de mercurio inorgánico por bacterias orales es aproximadamente 2-3 $\mu \mathrm{g} / \mathrm{día}^{(14)}$.

Niveles elevados de mercurio placentarios se han reportado en trabajadores del equipo odontológico que, durante todo el embarazo, fueron expuestos al vapor de mercurio $\left(\mathrm{Hg}^{0}\right)$ liberados durante la preparación de la amalgama en los consultorios dentales, la transferencia 
del mercurio de las amalgamas, de la madre al feto, ha sido reportado en muestras de necropsias humanas; niveles elevados de mercurio total se han observado en el cerebro, el hígado y los riñones de fetos humanos (21). Esta última información tiene relevancia local ya que se ha demostrado hace una década que existen niveles elevados de vapor de mercurio (>50 $\mu \mathrm{g} / \mathrm{m} 3)$ en el ambiente laboral de una clínica universitaria cuyo valor supera los máximos permitidos de $25 \mu \mathrm{g} / \mathrm{m3}{ }^{(22)}$ y de mercurio en orina (promedio $32 \mu \mathrm{g} / \mathrm{L}$ ) en el personal que labora en dicha clínica ${ }^{(23)}$.

Un estudio determinó una fuerte correlación positiva (rho $=0,79$ ) entre los niveles de mercurio en la sangre materna y del cordón umbilical. Los niveles de $\mathrm{Hg}$ en la sangre del cordón umbilical se asociaron significativamente con el número de amalgamas maternas. Los valores promedio de las concentraciones de $\mathrm{Hg}$ fueron $0,63 \mu \mathrm{g} / \mathrm{L}$ (rango de 0,14 a $2,9 \mu \mathrm{g} / \mathrm{L}$ ) y $0,80 \mu \mathrm{g} / \mathrm{L}$ (rango 0,15 a $2,54 \mu \mathrm{g} / \mathrm{L}$ ) de sangre materna y del cordón, respectivamente. Sin embargo, ninguna de las concentraciones de mercurio en la sangre del cordón alcanza el nivel que se considera peligroso para efectos del desarrollo neurológico en los niños expuestos a $\mathrm{Hg}$ en el útero (dosis de referencia de la EPA para el $\mathrm{Hg}$ de 5,8 $\mu \mathrm{g} / \mathrm{L}$ en sangre del cordón umbilical) ${ }^{(6)}$

Por otro lado, los bebes de gestantes con $\geq 6$ amalgamas fueron 3,2 veces más propensos a ser diagnosticados con autismo (severo) en comparación al trastorno del espectro autista leve de los bebes de gestantes con $\leq$ 5 amalgamas. Todo lo expuesto exige tomar medidas como políticas públicas sobre la amalgama dental, que deben considerar la exposición al Hg en mujeres antes y durante la edad fértil y la posibilidad de exposición fetal posterior y los resultados adversos ${ }^{(7)}$.

Faustman y otros investigadores informaron sobre los efectos del mercurio en el desarrollo neuronal, declaró: "La exposición al mercurio alteró el número de células y la división celular; estos impactos se han postulado como modos de acción de los efectos adversos observados en el desarrollo neuronal. Las posibles implicaciones de estas observaciones son evidentes cuando se evalúa en el contexto de investigaciones que muestran que la proliferación celular alterada y efectos neuropatológicos focales se han relacionado con los déficits neuroconductuales específicos (por ejemplo, autismo)" (7).

El mecanismo subyacente de las observaciones mencionadas derivan del hecho que los iones mercurio tienen elevada afinidad por grupos sulfidrilo de aminoácidos y, de esta forma, se bloquean las enzimas ${ }^{24)}$. Asimismo, se ha demostrado que niveles bajos de metilmercurio afectan primariamente la citoarquitectura a partir del sistema microtubular. Los microtúbulos intactos son requeridos para la división y migración celular. Los microtúbulos son formados en un proceso de ensamblado de los monómeros de tubulina alfa y beta. Aparentemente, el metilmercurio se une a los ligandos tiol (-SH) en los monómeros de tubulina y bloquea el proceso de ensamblaje ${ }^{(25-}$ 27). Se informó también que los niveles de glutatión, una sustancia producida por el cuerpo que es muy importante para la desintoxicación de metales pesados, por su polimorfismo puede afectar el metabolismo del mercurio ${ }^{28)}$. Es así que se encontraron disminuidos de manera significativa a través del espectro del autismo. Estos investigadores reportaron que sus observaciones sugieren que los niveles de glutatión cumplen un papel funcional importante en ayudar a dictar la severidad del autismo tras la intoxicación por mercurio ${ }^{(7)}$.

Además, la OMS ${ }^{(29)}$ indica que el número de superficies dentales de amalgama y los niveles de mercurio en cerebro de necropsias están relacionados, y que en algunas exposiciones al mercurio debido a la amalgama dental, a pesar que la concentración en el cerebro es baja, pueden desarrollarse eventos adversos, principalmente en individuos genéticamente susceptibles ${ }^{(30-32)}$, sobre todo cuando se ha demostrado que las concentraciones de mercurio en orina podrían no reflejar la retención tisular de mercurio en los tejidos más sensibles como el cerebro y las glándulas endocrinas ${ }^{(4,33)}$. Finalmente, la "Colaboración en Materia de Salud y el Aprendizaje de Medio Ambiente y Discapacidades del Desarrollo" (2008) publicó una declaración de consenso informando que la exposición a mercurio puede producir trastornos del espectro autista ${ }^{(7)}$.

\section{DESCARGAS DE MERCURIO DESDE LOS CONSULTORIOS ODONTOLÓGICOS AL ALCANTARILLADO Y EL MEDIOAMBIENTE}

La investigación realizada por Stone registró por primera vez el MeHg en aguas residuales de unidades dentales, este informe establece la existencia de especies de $\mathrm{Hg}$ biodisponibles en el efluente de aguas residuales de las instalaciones de tratamiento dental. La cantidad de $\mathrm{MeHg}$ es bajo en comparación con $\mathrm{Hg}$ total, sin embargo, cabe destacarlo cuando se considera la toxicidad del $\mathrm{MeHg}$; además, la concentración de $\mathrm{MeHg}$ en muestras de aguas residuales dentales es varias órdenes de magnitud superior que en las muestras ambientales ${ }^{(34)}$. Como se mencionó anteriormente están correlacionados los niveles de $\mathrm{MeHg}$ en boca con el número de restauraciones que contienen $\mathrm{Hg}$, lo que sugiere que la metilación puede 
ocurrir en la cavidad oral. Las BRS en la cavidad oral de los pacientes pueden proporcionar un mecanismo por el cual las aguas residuales de la unidad dental se 'inoculan' con estas bacterias ${ }^{(20,34,35)}$.

La mayor parte del mercurio en las aguas residuales del consultorio dental se encuentra en forma de amalgama dental que es capturado por los sistemas de tratamiento de aguas residuales denominados obras de tratamiento de propiedad pública (OTPP) en países desarrollados. Sin embargo, el mercurio disuelto, que la EPA define como el mercurio que puede pasar a través de un filtro de 0,45 micrómetros, es demasiado pequeño para ser capturado por las OTPP. Como resultado, el mercurio disuelto, a menudo aparece en el efluente de la estación de depuración u OTPP. Estas descargas de mercurio también están asociadas con desinfectantes que contienen agentes oxidantes que liberan mercurio de la amalgama al interaccionar con esta en los tubos de desagüe de los consultorios dentales. En estudios in vitro, se informó que las soluciones de hipoclorito así como desinfectantes que contienen cloro, bromo, yodo, peróxido y algunos compuestos fenólicos liberan más mercurio a partir de partículas de amalgama de lo que hizo el control (agua). Se demostró que el pH no es un buen predictor de la liberación de mercurio de la amalgama ya que las seis preparaciones que liberan más mercurio de la amalgama (aproximadamente de 17 a 340 veces) que el agua desionizada eran o muy ácidos ( $\mathrm{pH} \mathrm{1,76} \mathrm{a} \mathrm{2,59)}$ o muy alcalinos $\left(\mathrm{pH} 10,72\right.$ a 12,35) ${ }^{(36,37)}$.

El cloro tiene una larga historia en la desinfección del agua potable, con el primer uso registrado en 1896. Recientemente, ha sido objeto de escrutinio debido a sus derivados como los trihalometanos (THM) y los ácidos haloacéticos (HAA) - producidos cuando las aguas superficiales se desinfectan con cloro, los cuales se cree que son cancerígenos. En EE.UU., en un esfuerzo por cumplir con las nuevas regulaciones de agua potable, muchos servicios de tratamientos del agua han comenzado a cambiar a cloraminación debido a la tendencia disminuida de la cloramina para producir subproductos halogenados. Es así que, un estudio evaluó el nivel de mercurio liberado a partir de la amalgama, al ser expuesto a varias concentraciones de cloro y cloramina por separado, y se determinó que había una diferencia significativa entre las mediciones de mercurio a la exposición de $10 \mathrm{mg} / \mathrm{L}$ de cloro en comparación con $10 \mathrm{mg} / \mathrm{L}$ de cloramina, con liberaciones de mercurio de $0,59 \mathrm{mg} / \mathrm{L}$ y $0,023 \mathrm{mg} / \mathrm{L}$, respectivamente. Pero existe una equivalencia en liberación de mercurio cuando ambas sustancias halógenas no superan $1 \mathrm{mg} / \mathrm{L}^{(38)}$.

Dar a conocer el riesgo de efectos tóxicos causados por la acumulación de mercurio antropogénico en los ecosistemas ha resultado en una creciente presión para reducir la descarga de desechos de mercurio. En consecuencia, se viene dando una mayor atención a los problemas de los desechos de mercurio de las clínicas dentales. Asimismo, se han establecido en varios países, restricciones a la manipulación y la descarga de residuos contaminados ${ }^{(39)}$. La instalación de un aparato de separación de amalgama certificada en clínicas dentales es obligatoria desde hace más de 20 años en varios países, como por ejemplo, Suiza, Alemania, Suecia y Dinamarca. La aprobación de separadores de amalgama incluye exámenes clínicos o de laboratorio que exigen el 95-99\% de eficiencia de separación ${ }^{(39,40)}$. Una investigación en Arabia determinó que las descargas de mercurio al alcantarillado, por un total de 330 unidades dentales y 155 operadores, fue de 437,7 mg de mercurio en dos meses, lo cual, según cálculos efectuados, sobrepasa excesivamente los valores máximos permisibles locales en las descargas de mercurio al desagüe que es de $50 \mathrm{ug} / \mathrm{L}^{(41)}$.

En una investigación más reciente realizada en Manaos (Brasil), los análisis de los sedimentosquecontenían residuos hospitalarios detectaron una concentración de mercurio de $2683 \mathrm{ug} \mathrm{Hg/g}$, que es 5,5 veces mayor que la concentración de nivel umbral que puede causar daños adversos a la biota; una cifra que es similar a las concentraciones encontradas en muchas minas de minerales. También se determinó que existen clínicas dentales en la ciudad que desechan los residuos de mercurio en el sistema de eliminación de residuos comunes, lo que contraviene las normas de seguridad sanitaria locales en Manaos. En este caso, sobre todo en los países en desarrollo, la mayoría de los residuos de amalgama son irregularmente transportados a vertederos desde la ciudad, donde pueden contaminar el suelo, los ríos y las aguas subterráneas ${ }^{(42)}$.

Actualmente hay quienes argumentan que: "Como una aleación, la amalgama de desecho es física y químicamente estable y no se descompone fácilmente en sus componentes" (43). Sin embargo, la amalgama se clasifica como un compuesto intermetálico, porque se mezcla con plata, estaño, cobre, y zinc. Desde el punto de vista químico, este compuesto intermetálico es inestable por definición, debido a filtraciones de vapor de mercurio de la amalgama dental en el tiempo (16). Además de las numerosas investigaciones que demuestran que las partículas de amalgama en el alcantarillado liberan $\mathrm{Hg}$ al ser expuestos a agentes oxidantes como desinfectantes y limpiadores químicos, como se mencionó anteriormente ${ }^{(34,36-38)}$.

Por otra parte, de unas 50000 incineraciones, se ha estimado aproximadamente que $170-180 \mathrm{~kg}$ de mercurio metálico se emite a la atmósfera. La mayoría de los 
crematorios modernos tienen filtros de chimenea a base de selenio que disminuyen significativamente la cantidad de vapor de mercurio que se libera a la atmósfera. Sin embargo, el efecto ambiental de la cremación de las amalgamas de los cadáveres no puede estimarse hasta que se lleve a cabo un estudio mundial sobre este origen de mercurio ${ }^{(5,43)}$. En cumplimiento de estos requerimientos, mediciones de mercurio liberado a la atmosfera durante cremaciones realizadas en Japón, país con el porcentaje más alto de incineraciones en el mundo, con un $99,9 \%$ del total de cadáveres cremados en el 2007, demostraron mediante estimaciones un total de emisiones de mercurio de $35,1 \mathrm{~kg} /$ año y con una proyección de 2,6 veces este valor para el $2037^{(44)}$.

La asociación entre la liberación del Hg elemental al medioambiente y su conversión en $\mathrm{MeHg}$ ha sido de gran interés para regular las emisiones, asimismo, es de constante preocupación en salud pública debido a las repercusiones en la salud de la población, es así que los sitios de metilación biológica del mercurio en ríos, lagos y mares contaminados han sido de interés. La mayoría de los estudios han concluido que la metilación se produjo en el sedimento ${ }^{(45)}$. La preocupación se fundamenta en que se ha informado que, por lo general, de 90 a $99 \%$ del mercurio total en el medioambiente está asociado con el sedimento, y menos del 1\% se acumula en la biota. En contraste, 90 a $99 \%$ de $\mathrm{MeHg}$ se acumula en la biota y 1 a $10 \%$ encontrado en el sedimento. Por lo tanto, es obvio que el $\mathrm{MeHg}$ es la principal especie de $\mathrm{Hg}$ que causa más preocupación sobre la exposición humana ${ }^{(46)}$. El mercurio se acumula en la cadena alimentaria, presenta un alto grado en el medio acuático, con los niveles más altos encontrado en los depredadores. Se ha descrito una magnificación biológica o bioamplificación de hasta 100000 veces desde el nivel de las algas a los depredadores ${ }^{(39,43)}$.

La disponibilidad neta de metilmercurio está regulada por las tasas relativas de su producción y descomposición (es decir, desmetilación). Por lo tanto, los mecanismos bacterianos de metilación y desmetilación han sido de interés, en tanto, los estudios ambientales sobre el ciclo del mercurio se han centrado en la importancia relativa de estas dos reacciones competitivas en los ecosistemas acuáticos ${ }^{(47)}$. Sin embargo, la tasa de metilación frente a la desmetilación, en la práctica, es abrumadoramente desproporcionada y lo ejemplifica la mayor tragedia de contaminación por MeHg: la enfermedad de Minamata (EM), que es el envenenamiento por $\mathrm{MeHg}$ que se produjo en los seres humanos que ingirieron pescado y mariscos contaminados por $\mathrm{MeHg}$, a partir de la liberación de mercurio elemental e inorgánico descargado en las aguas residuales de una planta química (Chisso Co. Ltd.) en $1956^{(48,49)}$.

\section{MEDIDAS DE RESPONSABILIDAD Y VALORES ETTICOS DE LA PROFESIÓN: MEJORES PRÁCTICAS DE MANEJO}

Desde un punto de vista clínico restaurador, la amalgama dental continúa siendo un excelente material de obturación en el sector posterior $(50,51)$. Pero, debido a la creciente preocupación por el mercurio como químico persistente, bioacumulativo y tóxico (PBT), el uso de mercurio en muchas industrias y productos en países desarrollados como los Estados Unidos ha disminuido considerablemente desde principios de $1980{ }^{(52)}$. Aunque los dentistas individualmente generan pequeñas cantidades de desechos perjudiciales para el medioambiente, los residuos acumulados producidos por la profesión pueden tener un significativo impacto ambiental ${ }^{(53)}$. A nivel global casi 3800 toneladas de mercurio son empleadas anualmente para uso antropogénico, de los cuales, se ha estimado que el $6-8 \%$ se utiliza en odontología. Alrededor de 2000 toneladas es la emisión global de mercurio que se vierten anualmente en el medioambiente como resultado de las actividades humanas, tales como el procesamiento de minerales y la combustión de combustibles fósiles ${ }^{(5)}$ (Figura 1).

Consumo de mercurio, toneladas

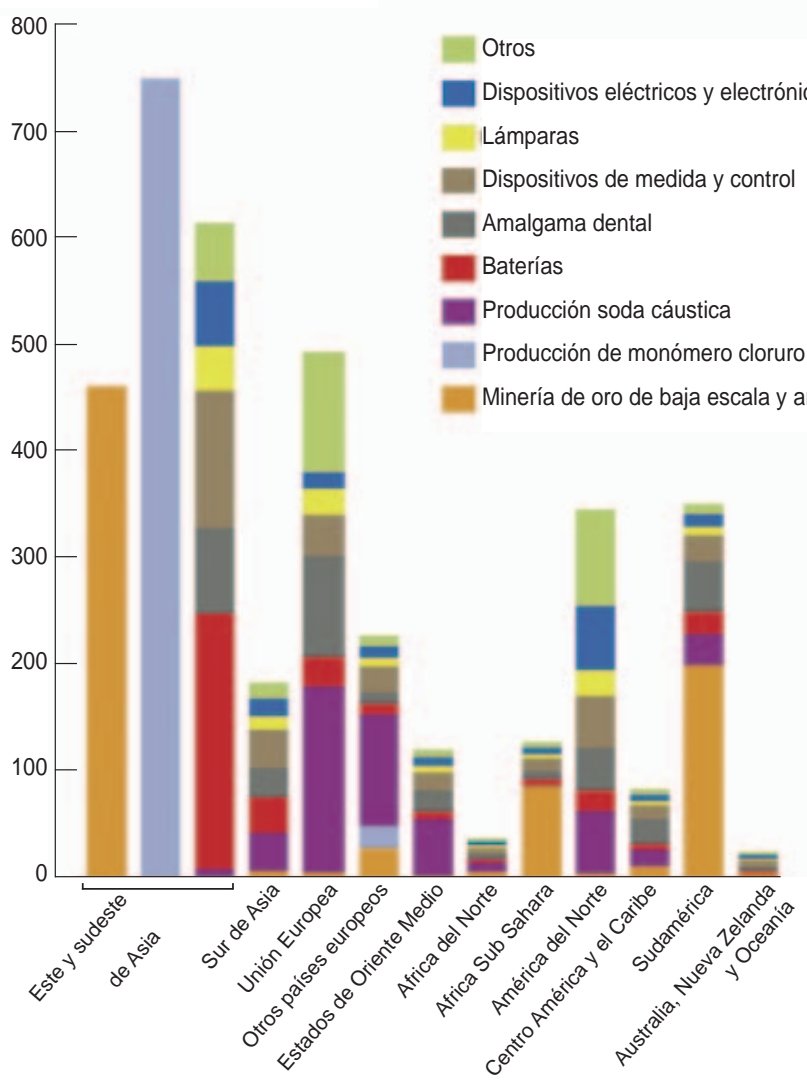

Figura 1. Consumo global de mercurio en el año 2005 (3798 toneladas) por aplicación y por región (AMAP/UNEP, 2008) ${ }^{(5)}$ 
Dentro de los países que adoptaron medidas de salud pública con respecto a la amalgama dental tenemos a Noruega, Dinamarca y Suecia, quienes prohibieron su uso en el 2008. Simultáneamente, Canadá y Alemania recomiendan evitar la colocación y remoción de amalgamas durante la gestación, así como a pacientes con problemas renales ${ }^{(31,54)}$. Francia, Finlandia y Austria han recomendado que se use materiales dentales alternativos cuando se trate de mujeres embarazadas (15). Mientras que en Alemania, además de estas últimas medidas, no está permitido la colocación de amalgamas en niños, por el principio de precaución, desde $1997{ }^{(24)}$. Se alienta a los profesionales a seguir las mejores prácticas de manejo (MPM) en el empleo y eliminación de la amalgama dental, con el propósito de limitar sus posibles efectos ambientales. Las mejores prácticas de manejo se aplican a una variedad de desechos peligrosos y dependen del tipo de residuos en cuestión. Están diseñados para proporcionar directrices a los médicos y así limitar los riesgos ocupacionales y ambientales de una determinada substancia ${ }^{(53,55)}$.

Dentro de las medidas adoptadas como MPM se encuentran: el empleo de aleación y mercurio preencapsulados; el reciclaje de la amalgama a todo nivel de procedimientos; no emplear ni verter desinfectantes que contengan hipoclorito de sodio, cloro, yodo y peróxidos de hidrógeno, entre otros, a los desagües de la unidad dental y del consultorio en general, y el empleo de separadores de amalgama ${ }^{(55)}$. Como profesionales de la salud somos legalmente responsables del recojo, almacenamiento y envió a empresas especializadas para reciclado de los residuos, tanto de partículas de amalgama grandes y finas. Los separadores de amalgama ISO 11143 certificados, son capaces de reducir las partículas de amalgama dental en las aguas residuales en más de un $95 \%{ }^{(53,56,57)}$.

Todavía se sostiene que la amalgama dental continúa siendo el material más utilizado como restaurador dental, ya que las restauraciones de amalgama son duraderas y de "bajo costo" (3), pero cuando se confronta con la necesidad de emplear sustancias tóxicas con responsabilidad, ello demanda evaluaciones costoefectividad, como se tiene a continuación: un estudio determinó el costo-efectividad del empleo de separadores de amalgama en las clínicas dentales de EE.UU., donde se evaluó la descarga de mercurio de las instalaciones dentales en forma de amalgama a las aguas superficiales. El uso anual de mercurio en forma de amalgama en los EE.UU. es de aproximadamente 31,9 toneladas métricas. Se estima que 26,9 toneladas métricas de mercurio en forma de amalgama se descargan anualmente a los sistemas de aguas residuales internas de instalaciones dentales durante la colocación y remoción de amalgamas.
El análisis de costo efectividad determinó que el costo anual para la industria dental para reducir los vertidos de mercurio a través de la utilización de separadores de amalgama oscilaría entre \$380 millones a \$1140 millones por tonelada ${ }^{(52)}$.

Una vez evaluados todos los aspectos implícitos en el empleo responsable de sustancias toxicas por medio del empleo de separadores de amalgama, que es uno de los lineamientos de las MPM, la denominación de "bajo costo" de las amalgamas no se ajusta a la verdad ${ }^{(4,5)}$. En países desarrollados como Canadá, un memorando de entendimiento entre la Asociación Dental Canadiense (CDA) y el gobierno, tuvo como objetivo reducir la liberación de mercurio de las amalgamas dentales en un 95\% a partir del 2005. Este esfuerzo voluntario alentado por la CDA está destinado a promover altos estándares morales y éticos dentro de la profesión odontológica en relación con la problemática de la amalgama dental. Para lograr la reducción del 95\% se alienta una incorporación de las mejores prácticas de manejo, así como la instalación de trampas en la unidad, filtros de vacío y separadores de amalgama con certificación ISO $11143^{(53)}$.

Mientras que en países en desarrollo, se ha podido determinar que la amalgama dental tiene un gran impacto en los residuos sólidos urbanos, no solo en términos de la cantidad generada, sino también debido al riesgo potencial que representa para la salud pública y el medioambiente. Algunos de los principales obstáculos para la prevención y el control de los problemas ambientales causados por la falta de gestión de residuos son el desinterés político, la legislación inadecuada y la falta de recursos humanos, financieros y de información (42). Debe producirse una reflexión en el personal odontológico y administrativo de las instituciones prestadoras de servicios en salud oral, las entidades de vigilancia y control y las instituciones académicas formadoras del talento humano, con el fin de implementar acciones integrales y metodologías seguras a corto plazo que generen un servicio de mejor calidad y mínimo riesgo_para el personal laboralmente expuesto y para el ecosistema. Lamentablemente, no existe conciencia con respecto a las mediciones ambientales de mercurio en la gran mayoría de países en desarrollo ${ }^{(58)}$.

Finalmente, en octubre de 2013, en el Convenio de Minamata sobre el Mercurio, se abordó la preocupación en relación con el potencial de riesgo para la salud humana y el daño ambiental de las emisiones intencionales de mercurio; los países adherentes al convenio, entre ellos el Perú, acordaron reducir de forma gradual su empleo en la industria, pero con respecto a la amalgama dental, no se exigieron objetivos vinculantes ni medibles para alcanzar estos objetivos ${ }^{(59)}$. 


\section{CONCLUSIONES}

En pacientes embarazadas no se les debe colocar ni remover amalgamas dentales por el peligro de aumentar la exposición a niveles altos de mercurio al feto. Las autoridades sanitarias deberían proveer regulaciones respecto a la eliminación de desechos de amalgama que eviten la contaminación del medioambiente. Las mejores prácticas manejo deben ser aplicadas a corto plazo por el integro de la profesión odontológica, incluyendo las instituciones académicas.

Fuentes de financiamiento: autofinanciado.

Conflictos de interés: el autor declara no tener conflictos de interés.

\section{REFERENCIAS BIBLIOGRÁFICAS}

1. Lorscheider FL, Vimy MJ, Summers AO. Mercury exposure from "silver" tooth fillings: emerging evidence questions a traditional dental paradigm. FASEB J. 1995 Apr;9(7):504-8.

2. Esteban-Vasallo MD, Aragonés N, Pollan M, López-Abente G, Perez-Gomez B. Mercury, cadmium, and lead levels in human placenta: a systematic review. Environ Health Perspect. 2012 Oct;120(10):1369-77. doi: 10.1289/ ehp.1204952.

3. Mutis MJ, Pinzón JC, Castro G. Las amalgamas dentales: ¿un problema de salud pública y ambiental? Revisión de la literatura. Univ Odontol. $2011 \mathrm{Jul}-$ Dic;30(65):63-70.

4. Mutter J, Naumann J, Guethlin C. Comments on the Article "The Toxicology of Mercury and Its Chemical Compounds" by Clarkson and Magos (2006). Crit Rev Toxicol. 2007;37(6):537-49.

5. Pacyna EG, Pacyna JM, Sundseth K, Munthe J, Kindbom K, Wilson S, et al. Global emission of mercury to the atmosphere from anthropogenic sources in 2005 and projections to 2020. Atmospheric Environment. 2010 Jun;44(2010):2487-99.

6. Palkovicova L, Ursinyova M, Masanova V, Yu Z, Hertz-Picciotto I. Maternal amalgam dental fillings as the source of mercury exposure in developing fetus and newborn. J Expo Sci Environ Epidemiol. 2008 May;18(3):326-31.

7. Geier DA, Kern JK, Geier MR. A prospective study of prenatal mercury exposure from maternal dental amalgams and autism severity. Acta Neurobiol Exp (Wars). 2009;69(2):189-97.

8. Caserta D, Graziano A, Lo Monte G, Bordi G, Moscarini M. Heavy metals and placental fetal-maternal barrier: a mini-review on the major concerns. Eur Rev Med Pharmacol Sci. 2013 Aug;17(16):2198-206.
9. Guzzi G, Pigatto PD. Occupational exposure to mercury from amalgams during pregnancy. Occup Environ Med. 2007 Oct;64(10):715-6.

10. Barregard L, Trachtenberg F, McKinlay $S$. Renal effects of dental amalgam in children: the New England children's amalgam trial. Environ Health Perspect. 2008 Mar;116(3):394-9. doi: 10.1289/ ehp.10504.

11. Bellinger DC, Daniel D, Trachtenberg F, Tavares M, McKinlay S. Dental amalgam restorations and children's neuropsychological function: the New England Children's Amalgam Trial. Environ Health Perspect. 2007 Mar;115(3):440-6.

12. Melchart D, Kohler W, Linde K, Zilker T, Kremers L, Saller R, et al. Biomonitoring of mercury in patients with complaints attributed to dental amalgam, healthy amalgam bearers, and amalgamfree subjects: a diagnostic study. Clin Toxicol (Phila). 2008 Feb;46(2):133-40. doi: 10.1080/15563650701324211.

13. Björnberg KA, Vahter M, Berglund B, Niklasson B, Blennow M, SandborghEnglund G. Transport of methylmercury and inorganic mercury to the fetus and breast-fed infant. Environ Health Perspect. 2005 Oct;113(10):1381-5.

14. Guzzi G, Minoia C, Pigatto PD, Severi G. Methylmercury, amalgams, and children's health. Environ Health Perspect. 2006 Mar;114(3):A149; author reply A149-50.

15. Dental amalgam: update on safety concerns. ADA council on Scientific Affairs. J Am Dent Assoc. 1998 Apr;129(4):494503.

16. Guzzi G, Grandi M, Cattaneo C. Should amalgam fillings be removed?. Lancet. 2002 Dec 21-28;360(9350):2081

17. Barregård L, Sällsten G, Järvholm B. People with high mercury uptake from their own dental amalgam fillings. Occup Environ Med. 1995 Feb;52(2):124-8.
18. Torres MA, Irribarra R, Ortega A, Romo F, Campos FO. Riesgos de intoxicación con biomateriales en odontología. Primera parte. Rev Dental Chile. 2002;93(3):17-22.

19. Sandborgh-Englund G, Einarsson C, Sandström M, Ekstrand J. Gastrointestinal absorption of metallic mercury. Arch Environ Health. 2004 Sep;59(9):449-54.

20.Langendijk PS, Kulik EM, Sandmeier $\mathrm{H}$, Meyer J, van der Hoeven JS. Isolation of Desulfomicrobium orale sp. nov. and Desulfovibrio strain NY682, oral sulfate-reducing bacteria involved in human periodontal disease. Int J Syst Evol Microbiol. 2001 May;51(Pt 3):1035-44.

21. Pigatto PD, Minoia C, Ronchi A, Guzzi G. Human placenta and markers of heavy metals exposure. Environ Health Perspect. 2013 Jan;121(1):A10. doi: 10.1289/ehp.1206061.

22. Ramírez AV. Intoxicación ocupacional por mercurio. An Fac med. 2008;69(1):46-51.

23. Perales Zamora S, Madrid MT, Alarcón WA, Echeandia J, Chein S. Niveles de mercurio en orina de profesores, alumnos y técnicos de la clínica odontológica de la Facultad de Odontología de la Universidad Nacional Mayor de San Marcos. Odontologia Sanmarquina. 2003;6(12):16-20.

24. Bose-O’Reilly S, McCarty KM, Steckling $\mathrm{N}$, Lettmeier B. Mercury exposure and children's health. Curr Probl Pediatr Adolesc Health Care. 2010 Sep;40(8):186215. doi: 10.1016/j.cppeds.2010.07.002.

25. Clarkson TW, Magos L. The toxicology of mercury and Its chemical compounds. Crit Rev Toxicol. 2006 Sep;36(8):609-62.

26. Clarkson TW, Magos L, Myers GJ. The toxicology of mercury--current exposures and clinical manifestations. $\mathrm{N}$ Engl J Med. 2003;349(18):1731-7.

27. Clarkson TW. The three modern faces of mercury. Environ Health Perspect. 2002 Feb;110 Suppl 1:11-23. 
28. Gundacker C, Gencik M, Hengstschläger $\mathrm{M}$. The relevance of the individual genetic background for the toxicokinetics of two significant neurodevelopmental toxicants: mercury and lead. Mutat Res. 2010 Oct;705(2):130-40. doi: 10.1016/j. mrrev.2010.06.003.

29. World Health Organization. The hazards of mercury. IPCS News. 1992 March;1:4-5.

30. Koral SM. Mercury from dental amalgam: exposure and risk assessment. Compend Contin Educ Dent. 2013 Feb;34(2):138-40, 142, 144 passim.

31. Trzcinka-Ochocka M, Gazewski A, Brodzka A. Exposure to mercury vapors in dental workers in Poland. Int J Occup Med Environ Health. 2007;20(2):14753.

32. Guzzi G, Grandi M, Cattaneo C, Calza S, Minoia C, Ronchi A, et al. Dental amalgam and mercury levels in autopsy tissues: food for thought. Am J Forensic Med Pathol. 2006 Mar;27(1):42-5.

33. Guzzi G, Pigatto PD. Urinary mercury levels in children with amalgam fillings. Environ Health Perspect. 2008 Jul;116(7):A286-A287. doi: 10.1289/ ehp. 11235 .

34. Stone ME, Cohen ME, Liang L, Pang P. Determination of methyl mercury in dental-unit wastewater. Dent Mater. 2003 Nov;19(7):675-9.

35.Environmental Protection Agency. Health Services Industry Detailed Study Dental Amalgam 2008 [internet]. Washington, DC: EPA; 2008 [citado el 05 enero de 2014]. Disponible en: http://water.epa.gov/scitech/wastetech/ guide/304m/upload/2008_09_08_ guide_304m_2008_hsi-dental-200809. pdf

36. Batchu H, Hwai-Nan C, Rakowski D, Fan PL. The effect of disinfectants and line cleaners on the release of mercury from amalgam. JADA. 2006;137(10):141925.

37. Stone ME, Kuehne JC, Cohen ME, Talbott JL, Scott JW. Effect of iodine on mercury concentrations in dentalunit wastewater. Dent Mater. 2006 Feb;22(2):119-24.

38. Stone ME, Scott JW, Schultz ST, Berry DL, Wilcoxon M, Piwoni M, et al. Comparison of chlorine and chloramine in the release of mercury from dental amalgam. Sci Total Environ. 2009 Jan 1;407(2):770-5. doi: 10.1016/j.scitotenv.2008.09.041.
39. Arenholt-Bindsley D. Dental amalgamenvironmental aspects. Adv Dent Res. 1992 Sep;6:125-30.

40. Adegbembo AO, Watson PA, Lugowski SJ. The weight of wastes generated by removal of dental amalgam restorations and the concentration of mercury in dental wastewater. J Can Dent Assoc. 2002 Oct;68(9):553-8.

41. Shraim A, Alsuhaimi A, Al-Thakafy JT. Dental clinics: a point pollution source, not only of mercury but also of other amalgam constituents. Chemosphere. 2011 Aug;84(8):1133-9. doi: 10.1016/j. chemosphere.2011.04.034.

42. de Souza JP, Nozawa SR, Honda RT. Improper waste disposal of silver-mercury amalgam. Bull Environ Contam Toxicol. 2012 May;88(5):797-801. doi: 10.1007/ s00128-012-0554-6.

43. Chin G, Chong J, Kluczewska A, Lau A, Gorjy S, Tennant M. The environmental effects of dental amalgam. Aust Dent J. 2000 Dec;45(4):246-9.

44. Takaoka M, Oshita K, Takeda N, Morisawa S. Mercury emission from crematories in Japan. Atmos Chem Phys. 2010;10:3665-71.

45. Furutani A, Rudd JW. Measurement of mercury methylation in lake water and sediment samples. Appl Environ Microbiol. 1980 Oct;40(4):770-6.

46. Shao D, Kang Y, Wu S, Wong MH. Effects of sulfate reducing bacteria and sulfate concentrations on mercury methylation in freshwater sediments. Sci Total Environ. 2012 May 1;424:331-6. doi: 10.1016/j.scitotenv.2011.09.042.

47. Oremland RS, Culbertson CW, Winfrey MR. Methylmercury decomposition in sediments and bacterial cultures: involvement of methanogens and sulfate reducers in oxidative demethylation. Appl Environ Microbiol. 1991 Jan;57(1):130-7.

48. Hamdy MK, Noyes OR. Formation of methyl mercury by bacteria. Appl Microbiol. 1975 Sep;30(3):424-32.

49. Harada M. Minamata disease: methylmercury poisoning in Japan caused by environmental pollution. Crit Rev Toxicol. 1995;25(1):1-24.

50.Peraire Ardèvol M. Liberación de mercurio por parte de las obturaciones de amalgama dental: tipo, cantidad, método de determinación y posibles efectos adversos. RCOE. 2011;16(1):43-7.
51. Soares AC; Cavalheiro A. A review of amalgam and composite longevity of posterior restorations. Rev Port Estomatol Med Dent Cir Maxilofac. 2010;51:15564.

52. Vandeven JA, McGinnis SL. An assessment of mercury in the form of amalgam in dental waste water in the United States. Water Air and Soil Pollution. 2005;164:349-66.

53. Hiltz M. The environmental impact of dentistry. JCDA. 2007 Feb;73(1):59-64.

54.Edlich RF, Cochran AA, Cross CL, Wack CA, Long WB 3rd, Newkirk AT. Legislation and informed consent brochures for dental patients receiving amalgam restorations. Int $\mathrm{J}$ Toxicol. 2008 Jul-Aug;27(4):313-6. doi: $10.1080 / 10915810802366851$.

55.55. World Health Organization. Future use of materials for dental restoration: report of the meeting convened at WHO HQ [Internet]. Geneva, Switzerland: WHO; 2009 [citado el 05 de enero de 2014]. Disponible en: http://www. who.int/oral_health/publications/dental_material_2011.pdf

56. Fan PL, Batchu H, Chou HN, Gasparac W, Sandrik J, Meyer DM. Laboratory evaluation of amalgam separators. J Am Dent Assoc. 2002 May;133(5):577-84.

57. Trap L. Canada-wide Standards: A pollution prevention program for dental amalgam waste. J Can Dent Assoc. 2001 May;67:270-3.

58. Ruiz JA, Pérez JI, Gómez GJ, Carmona ME, Zapata LA, Carmona R. Riesgo en el manejo de la amalgama dental en las entidades odontológicas medianas y pequeñas en el departamento de Antioquia, Colombia. Rev Fac Nac Salud Publica. 2009;27(2):187-97.

59. Mackey TK, Contreras JT, Liang BA. The Minamata Convention on Mercury: attempting to address the global controversy of dental amalgam use and mercury waste disposal. Sci Total Environ. 2014 Feb 15;472:125-9. doi: 10.1016/j.scitotenv.2013.10.115.

Correspondencia: Christhian Méndez Visag Dirección: Calle Wagner 295 San Borja. Lima. Perú

Teléfono: 961820585

Correo electrónico:cmendez_1275@hotmail.com 